\title{
COGNITIVE REACTION TIME PERFORMANCE AND SUBJECTIVE DROWSINESS: A CRITICAL EVALUATION OF THE EFFECT OF WHOLE-BODY VIBRATIONS
}

\author{
Amzar A and Fadhilah MS \\ Aero-ergonomics Research Cluster, Malaysian Institute of Aviation Technology, Universiti Kuala Lumpur, Sepang, \\ Selangor, Malaysia \\ * Corresponding author: Amzar A \\ Email: mohdamzar@unikl.edu.my
}

\begin{abstract}
It is believed that exposure to whole-body vibration (WBV) may increase seated occupant drowsiness, and seated occupant drowsiness may contribute to vehicular accidents. Previous studies on driver comfort have indicated that long-term exposure to WBV may have an adverse effect on musculoskeletal disorders. However, the effects of WBV on seated occupants' drowsiness have been less rigorously studied. Thus, this study aims to investigate the association between exposure to WBV and drowsiness level. Laboratory experiments were designed and involved eighteen healthy male volunteers. Volunteers were exposed to random gaussian vibration for 20-minutes with the frequency between $1-15 \mathrm{~Hz}$. The transmitted vibration magnitude was adjusted for each volunteer to become $0.2 \mathrm{~ms}^{-2}$ for low vibration magnitude and $0.4 \mathrm{~ms}^{2}$ for medium vibration magnitude. Volunteers' vigilance was measured by the Psychomotor Vigilance Task (PVT) before and after the vibration exposure. The analyses revealed a substantial drop in volunteers' vigilance level after exposure to vibration and the effect was more pronounced in high vibration amplitude $0.4 \mathrm{~ms}^{-2}$. These findings suggested that exposure to vibration even as low as 20-minutes may attribute to the reduction of alertness level.
\end{abstract}

Keywords: Whole-body vibration, drowsiness, psychomotor vigilance test

\section{INTRODUCTION}

Over the last decades, researchers have focused on early prediction and assessment on the drowsiness level of seated occupants' drowsiness in a range of transportation vehicles. There are many factors leading to drowsiness, including influences from substances like alcohol as well as sleep deprivation ${ }^{1,2}$. In this regard, numerous national and international safety standards have been drafted which help describe the health risks of perceived vibration to the human body. These standards establish guidelines and thresholds, such as weighting filters, that can use to approximate the comfort of the human ride, however, how drowsiness is caused by the vibration of a vehicle is yet to investigated and understood. In this regard, drowsiness is a multifactorial aspect that influences not only the notions of quality perception (comfort) and judgment (safety) but also health as long-term exposure to vibration could lead to muscularskeletal injuries ${ }^{2}$. Past studies had predicted and developed an understanding of how the human body subjectively responds to vibrations ${ }^{3}$. However, there is still a limited quantitative study that investigated the influence of vibration on seated occupants' drowsiness level.

Studies had shown that drowsiness and prolonged sleep deprivation could lead to declining response speed (1/RT), increase in reaction time (RT) and the increase in the number of lapses (RT > 500 $\mathrm{ms})^{4-6}$, but the attempt to establish the relations between psychomotor deficit such as drowsiness through measuring the reaction time performances, has not yet been able to fully investigate the drowsiness caused by exposure to vehicle vibration. This particular study is done by referring to the ISO2631-1; aptly named "Evaluation of human exposure to whole-body vibration' weightings and test procedures"7 guidelines which stipulate that regardless of the frequency content, the fixed frequency weighted r.m.s value of vibration transmitted to the seated human occupants will show a constant subjective comfort rating, in order to conduct an objective and subjective measurement of human participants' drowsiness level based on human perceived vibration.

\section{METHODS}

The study involved eighteen healthy, randomly selected male university students, with the mean age of $23.0 \pm 1.3$ years. For this study, the mean value of the participants' BMI was $22.6(\mathrm{SD}=2.54)$ $\mathrm{kg} / \mathrm{m}^{2}$. This has been calculated from the mean values of their height and weight measurements, which were $168.2 \pm 4.0 \mathrm{~cm}$ and $69.3 \pm 9.88 \mathrm{~kg}$ respectively. Epworth Sleepiness Scale (ESS) was utilized for the detection of any possible abnormalities in sleepiness that any of the participants may have or suffer from. This method was carried out before the actual experiment ${ }^{8}$. A score of $>10$ indicates excessive sleepiness, and participants with this score were exempted from the experiment ${ }^{9}$. The participants were instructed to have enough sleep a day prior, and based on the results, the ESS scores generated showed a 
range of $0-24$. For the interpretation; any value below 7 would be determined as normal, moderate would be for values between 8 - 10, and any number between 11 - 15 would indicate elevated risks. As a result, any case of more than 16 would indicate severe excessive daytime sleepiness $(E D S)^{10}$, showing a high risk for them to fall asleep in a range of monotonous situations ${ }^{11}$.

The time was measured using the $P C$-based Psychomotor Vigilance Test (PVT-192: Ambulatory Monitoring Inc., Ardsley, New York) ${ }^{12}$. Researchers who utilize this test would need to be aware of the two specific conditions to assess sustained attention during the task. The conditions for the 10-minute visual reaction time task are before vibration exposure and after vibration exposure. For this task, every participant must click a mouse as fast as possible; this is to signify their response to the visual stimulus' appearance. The stimulus was in the form of a diode which emits a red light that displays time in mili-seconds. A visual stimulus would appear in 2-10 seconds variable intervals during each 10-minutes session. All three of the PVT performance metrics during each PVT condition were generated by the software. The performance metrics consist of a median \& mean of reaction time and a number of lapses. For a response to be valid, it should be $>100 \mathrm{~ms}$. It would be determined as a false signal whenever it shows $<100 \mathrm{~ms}^{5}$. The participants were exposed to a Gaussian random vibration, with 1-15 Hz frequency bandwidth, for 20-minutes. During vibration condition, the participants were asked to sit comfortably while their feet as firmly fixed at the footrest, their back on the backrest and hands on their lap. They were also asked to restrict any physical movement. The footrest was isolated from the vibration and is not connected to the vibration table and. The participants also endured similar experimental procedures during the no-vibration condition. Here, after the first PVT test was completed, the participants were asked to sit for 20-minutes and the second PVT test will be carried out straight away after 20minutes of sitting.

As mentioned in previous literature ${ }^{13}$, Karolinska Sleepiness Scale (KSS) was utilized to measure participants' sleepiness at several designated times; before they are exposed to vibration, every 5 -minute interval of vibration, and after exposed to vibration. The 9-indicator scale ranges from 1 extremely alert to 9 - very sleepy. The measurement process begins when a test leader would make the announcement verbally; during the entire process, both the leader and participants are prohibited from verbally talking to each other aside from emergencies.

This study utilizes a specifically-designed, experimental rig (real-life vehicle seat set up with adjustable headrest, affixed on an aluminium

table with four air mountings). With the rigid mass of the table eliminated by the air mountings, it's inclination angle is set vertically at $15^{\circ}$. To calculate the table's excitation input force before measuring the drowsiness levels, on one corner of the table was set with a vertical servo-controlled hydraulic actuator (5 kN). As mentioned previously, readings of the measurement were interpreted by referring to ISO-2631-1 (1997) ${ }^{7}$. By using the accelerometer pad, the measurement was conducted to modify the hydraulic input force required for every participant (Fig. 1). Each participant was instructed to maintain a regular sleep pattern a week prior to the experiment and to avoid any caffeine intake. The experiment started at $9.00 \mathrm{AM}$, and prior to the experiment, participants were assessed by using the Epworth Sleepiness Scale (ESS). Those with a score exceeding 10 will be exempted from the experiment ${ }^{8}$. To ensure minimal learning effects, every participant had the opportunity to undergo a practice session. During the practice, they had to cope with the following conditions:

- Low amplitude vibration r.m.s.

- Medium amplitude vibration $0.4 \mathrm{~ms}^{-2}$ r.m.s.

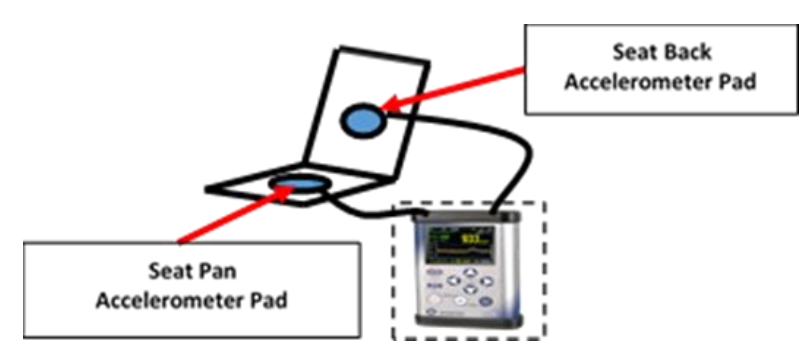

Figure 1: The vibration transmitted to the human body was measured by using two accelerometer pads that are connected to the seat back and seat pan measurement.

\section{RESULTS}

Table 1 presents the PVT results which measured the effects of vibration amplitude (ms $\mathrm{m}^{-2}$ r.m.s) on the participants' drowsiness level. Three PVT metrics were assessed, which are (1) minor lapse, (2) mean RT and (3) median RT. The readings between the PVT metrics (before and after the 20minute exposure to vibration) shows how significant $(p<0.05)$ the effects can affect their RT in both conditions. In addition, the participants have shown critical rises in the number of lapses and RT. The data in Table 1 shows that after a 20minute exposure to vibration, all of the participants showed increase in the average number of PVT lapses (mean \pm SEM) in both conditions participants from $(1.67 \pm 0.36$ to 3.67 $\pm 0.58, p=0.0018$ in low vibration amplitude and from $1.71 \pm 0.36$ to $4.86 \pm 0.46, p=0.0002$ in medium vibration amplitude which show a decrease in the level of alertness. The analysis showed different mean differences after medium 
vibration amplitude $\left(0.4 \mathrm{~ms}^{-2} \quad\right.$ r.m.s $)$ was compared to low vibration amplitude $\left(0.2 \mathrm{~ms}^{-2}\right.$ r.m.s). Such apparent changes demonstrate that the effect of vibration amplitude shows that the more pronounced lapse of attention in medium vibration amplitude $\left(0.4 \mathrm{~ms}^{-2}\right.$ r.m.s $)$, and the result is significant $(p=0.0024)$.

The mean RT for eighteen participants in both vibration conditions show statistical significance, and there is an increase in the reaction time participants. Due to exposure to vibration, the participants mean RT for the participants had increased from $(283.3 \pm 6.24$ to $320.2 \pm 10.68, p<$ 0.0001 in low vibration amplitude and from 281.1 \pm 6.56 to $360.6 \pm 11.50, \mathrm{p}<0.0001$ in medium vibration amplitude). Compared to low vibration amplitude $\left(0.2 \mathrm{~ms}^{-2}\right)$, the medium vibration amplitude $\left(0.4 \mathrm{~ms}^{-2}\right)$ had a bigger mean difference of mean RT. It signifies that the medium vibration amplitude $\left(0.4 \mathrm{~ms}^{-2}\right)$ significantly affects human drowsiness levels $(p=0.0027)$. Table 1 also indicates the increase of PVT metrics based on the median RT as a result of the exposure to low and medium vibration amplitude. In low vibration amplitude, their median RT increased from 268.5 \pm 7.21 to $301.3 \pm 9.68$ whilst their $p=0.002$. In medium vibration amplitude, their median RT also increased from $268.3 \pm 7.89$ to $329.1 \pm 10.13$ whilst their $\mathrm{p}<0.0001$.

The bigger difference in the mean for medium vibration amplitude $\left(0.4 \mathrm{~ms}^{-2}\right)$ shows that higher vibration amplitude has a more apparent effect of drowsiness on a human. Meanwhile, the effect sizes for all PVT metrics were measured and shown in Table 2. In this light, the magnitude of the reaction time changes between prior and after the exposure to vibration in both conditions is indicated by the increase of effect sizes. PVT metrics were arranged based on effect sizes and susceptibility to vibration. During the low vibration amplitude condition, the median RT indicated a high effect size $(E S=0.838)$ of the difference between prior and after 20-minutes of exposure to vibration. This shows higher susceptibility to vibration followed by mean RT $(E S=0.796)$ and minor Lapse $(E S=0.632)$. The moderate statistical and clinical differences between the two variables are evident when the effect sizes exceed 0.5 . Meanwhile, during the medium vibration amplitude condition, there was a high magnitude of the differences prior and after vibration exposure with the mean RT (ES = $0.848)$, followed by minor lapse $(E S=0.821)$ and median RT $(E S=0.784)$. Consequently, both results were compared, and the result shows the significant difference between low vibration amplitude conditions and medium vibration amplitude conditions, and the effect is more significant in medium vibration amplitude which shows higher effect sizes. Further analysis indicates that the mean RT is a sensitive PVT metrics in both vibration amplitude conditions study mentioned.

Table 1: A comparison of PVT metrics between low vibration amplitude and medium vibration amplitude is shown. A significant increase in reaction time and minor lapse ( $R T>500 \mathrm{~ms}$ ) can be observed following exposure to vibration. Medium amplitude vibration has proven to have more significant effects

\begin{tabular}{|c|c|c|}
\hline & \multicolumn{2}{|c|}{ Low vibration amplitude $0.2^{-2} \mathrm{rms}$} \\
\hline & Before exposure & After exposure \\
\hline Minor lapse $(n)$ & $1.67 \pm 0.36$ & $3.67 \pm 0.58$ \\
\hline Mean RT & $283.1 \pm 6.24$ & $320.2 \pm 10.68$ \\
\hline \multirow[t]{3}{*}{ Median RT } & $268.5 \pm 7.21$ & $301.3 \pm 9.68$ \\
\hline & \multicolumn{2}{|c|}{ Medium vibration amplitude $0.4^{-2} \mathrm{rms}$} \\
\hline & Before exposure & After exposure \\
\hline Minor lapse (n) & $\overline{1.71 \pm 0.36}$ & $4.86 \pm 0.46$ \\
\hline Mean RT & $281.1 \pm 6.56$ & $360.6 \pm 11.50$ \\
\hline Median RT & $268.3 \pm 7.89$ & $329.1 \pm 10.13$ \\
\hline
\end{tabular}

Table 2: The effect size of PVT metrics in both vibration conditions is shown. Bigger statistical and clinical differences between the two variables are influenced by the larger effect size.

\begin{tabular}{llc}
\hline & \multicolumn{2}{l}{ Low vibration amplitude of $0.2 \mathrm{~ms}^{-2} \mathrm{rms}$} \\
\hline Rank & PVT metrics & Effect size \\
$\mathbf{1}$ & Median RT & 0.838 \\
$\mathbf{2}$ & Mean RT & 0.796 \\
$\mathbf{3}$ & Minor lapse & 0.632 \\
& Medium vibration amplitude of $\mathbf{0 . 2} \mathrm{ms}^{-2} \mathrm{rms}$ \\
\multirow{2}{*}{ Rank } & PVT metrics & Effect size \\
$\mathbf{1}$ & Mean RT & 0.848 \\
$\mathbf{2}$ & Minor lapse & 0.796 \\
$\mathbf{3}$ & Median RT & 0.632 \\
\hline
\end{tabular}

Figure 2 shows the Karolinska Sleepiness Scale (KSS) plots as scores against time; for both low and medium vibration amplitude. Based on the repeated measures-ANOVA test $(p<0.0001)$ of all eighteen participants in both conditions, it can be observed that there are significant increases in KSS score after every subsequent 5-minutes of exposure to vibration compared to prior to exposure. Fig. 2 illustrate a definite decrease in the level of as reflected by the continuous increase in subjective sleepiness score during exposure to vibration in both vibration amplitude conditions $\left(0.2 \mathrm{~ms}^{-2}\right.$ r.m.s. and $0.4 \mathrm{~ms}^{-2}$ r.m.s. $)$.

The figure indicates that the average KSS score (mean \pm SEM) prior to vibration exposure for low and medium vibration amplitude was $2.56 \pm 0.16$ and $2.72 \pm 0.19$. There is no statistical significance difference $(p>0.05)$ between low and medium vibration amplitude conditions prior to vibration exposure. Meanwhile, being exposed to vibration 
for 10-minutes, the average KSS score for all participants had consistently increased to $5.56 \pm$ 0.35 (mean \pm SEM) in low vibration amplitude and $6.06 \pm 0.15$ (mean \pm SEM) in medium vibration amplitude. In this light there is only a slight difference between values obtained for low and medium vibration amplitude. $(p>0.05)$. From the figure, it can be observed that there is more pronounced drowsiness after the participants were exposed to the vibration for 20 minutes and the KSS values were $6.78 \pm 0.33$ (mean \pm SEM) in low vibration amplitude and $7.61 \pm 0.12$ (mean \pm SEM) in medium vibration amplitude. This clearly shows the depleting level of awareness in all participants after they were exposed to vibration for 20 minutes. One-way repeated measuresANOVA was carried out to investigate the statistical significance, and the results show highly significant intra-individual and interindividual differences for all participants $(p<$ 0.0001 ) for each vibration condition. In addition, it was observed that between low and medium vibration amplitude, there is no significant difference.

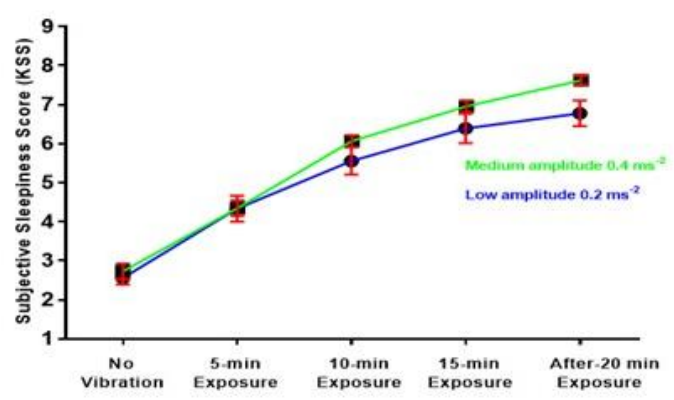

Figure 2: The mean of subjective sleepiness scores (KSS) for all the participants in two vibration conditions (low vibration amplitude and medium vibration amplitude)

\section{DISCUSSION}

This study hypothesized that when the participants are drowsy, they will not be able to provide good judgment on their drowsiness level. It proved that vibration has significantly affected human reaction time. How the drowsiness of seated humans can be affected by low vibration amplitude has also been further supported by previous literature ${ }^{14}$. These studies have shown a significant relationship between drowsiness level and vibration, as well as the significant increase in the reaction time after being exposed to vibration for 20 minutes ${ }^{15,16}$. This study's finding is parallel to Azizan et. $A l^{14}$ which postulated that the increase in vibration amplitude could more significantly impair the level of human alertness. The present study had compared low vibration amplitude $\left(0.2 \mathrm{~ms}^{-2}\right.$ r.m.s) with medium vibration amplitude $\left(0.4 \mathrm{~ms}^{-2} \mathrm{r} . \mathrm{m} . \mathrm{s}\right)$ and indicated that the degree of drowsiness, as measured by PVT, was more significant when vibration amplitude was double. In this regard, the increase in the transmitted vibration to the human body will make the drowsiness level more apparent. This finding shows that the link between exposure to vibration and the level of drowsiness. On the other hand, the study did not find any significant difference in the subjective measurement (KSS) in both vibration conditions. Aside from having similar findings that support previous literature ${ }^{17}$, this study has also generated important data on the effects of drowsiness contour developed which was caused by vibration. To conclude, more research is recommended to further strengthen the concepts and relationships pertaining to drowsiness levels and vibration exposure.

\section{CONCLUSION}

This present study is aimed to identify the impact of vibration on the reaction time and drowsiness level of seated occupants by conducting a comprehensive, objective measurement test (PVT) and subjective evaluation score (KSS). The finding supports the hypothesis that the human drowsiness level could be considerably influenced by the presence of low-frequency vibration (between $1-15 \mathrm{~Hz}$ ). The results show that compared to low vibration amplitude $\left(0.2 \mathrm{~ms}^{-2}\right.$ r.m.s), medium vibration amplitude $\left(0.4 \mathrm{~ms}^{-2}\right.$ r.m.s) study creates a higher level of perceived drowsiness among the participants. On the other hand, the KSS result shows that there is no significant difference subjective drowsiness level found in both conditions of vibration while the PVT test shows a substantial increase in the reaction time and the number of lapses in both conditions, and medium vibration amplitude shows a higher degree of error. As a conclusion, drowsiness levels increase with the influence of increasing vibration amplitude levels.

\section{ACKNOWLEDGEMENTS}

The authors wish to thank the staffs and facilities at RMIT University, Melbourne and Universiti Kuala Lumpur in providing assistance in this study.

\section{COMPETING INTERESTS}

There is no conflict of interest.

\section{REFERENCES}

1. Arnedt JT, Wilde GJ, Munt PW MacLean AW. 2001, How do prolonged wakefulness and alcohol compare in the decrements they produce on a simulated driving task. Accident; Analysis and Prevention. 33:337-44.

2. Philip P, Sagaspe P, Moore N, Taillard J, Charles A, Guilleminault C, Bioulac B. 2005, Fatigue, sleep restriction and driving performance. Accident; Analysis and Prevention. 37: 473-78. 
3. Azizan MA, Fard. M. 2013, Effects of vehicle seat dynamics on ride comfort assessment, in International Congress on Noise and Vibration.

4. Anderson C, Wales AWJ and Horne JA. 2010, PVT lapses and the eyes PVT lapses differ according to eyes open, closed, or looking away. Sleep. 33: 197-04.

5. Basner B and Dinges DF. 2011, Maximizing the sensitivity of the psychomotor vigilance test (PVT) to sleep loss. Sleep. 34: 581-91.

6. Jackson ML, Croft RJ, Kennedy GA, Owens K, and Howard ME. 2013, Cognitive components of simulated driving performance: Sleep loss effects and predictors. Accident; Analysis and Prevention.50: 438-44.

7. International Standard. ISO 2631-1 Mechanical vibration and shock.1997 Evaluation of human exposure to wholebody vibration.

8. John MW. 1991, A new method for measure daytime sleepiness the Epworth Sleepiness Scale. American Sleep Disorder Association and Sleep Research Society. 14: 540-45.

9. Shattuck NL and Matsangas P. 2015, Psychomotor vigilance performance predicted by Epworth Sleepiness Scale scores in an operational setting with the United States Navy. Journal of sleep research. 24: 178-80.

10. Karimi M, Hedner J, Lombardi C, Mcnicholas WT, Penzel T, Riha RL, Rodenstein D and Grote L. 2014, Driving habits and risk factors for traffic accidents among sleep apnea patients-a European multi-center cohort study. Journal of sleep research. 23: 689-99.

11. Howard ME, Desai AV, Grunstein RR, Hukins C, Armstrong JG, Joffe D, Swann P, Campbell DA and Pierce RJ. 2004, Sleepiness, sleep-disordered breathing, and accident risk factors in commercial vehicle drivers. American Journal of Respiratory and Critical Care Medicine. 170: 1014-21.

12. Khitrov MY, Laxminarayan S, Thorsley D, Ramakrishnan S, Rajaraman S, Wesensten NJ and Reifman J. 2014, PC-PVT: a platform for psychomotor vigilance task testing, analysis, and prediction. Behavior Research Methods. 46: 140-47.
13. Gillberg M, Kecklund G, and Akerstedt T.1994, Relations between performance and subjective ratings of sleepiness during a night awake. Sleep.17: 236-41.

14. Azizan MA, Fard M. 2014, The influence of vibrations on vehicle occupant fatigue, Internoise Conference.

15. Satou $Y$, Ando $H$, Nakiri $M$, Nagatomi $K$, Yamaguchi $Y$, Hoshino $M$, Tsuji $Y$, Muramoto J, Mori M, Hara $\mathrm{K}$ and Ishitake T. 2007, Effects of short-term exposure to whole-body vibration on wakefulness level. Industrial Health.45: 217-23.

16. Satou $Y$, Ishitake $T$, Ando $H$, Nagatomi $K$, Hoshiko M, Tsuji Y, Tamaki H, Shigemoto A, Kusano M, Mori M and Hara K. 2009, Effect of short-term exposure to wholebody vibration in humans: the relationship between wakefulness level and vibration frequencies. The Kurume Medical Journal. 56: 17-23.

17. Horne JA and Baulk SD 2004, Awareness of sleepiness when driving. Psychophysiology. 41: 161-65. 